\title{
microRNA-25 as a novel modulator of circadian Period2 gene oscillation
}

Inah Park', Doyeon Kim', Jeongah Kim², Sangwon Jang ${ }^{1}$, Mijung Choi ${ }^{1}$, Han Kyoung Choe ${ }^{1}$, Youngshik Choe ${ }^{3}$ and Kyungjin $\mathrm{Kim}^{1}$

\begin{abstract}
Circadian clock controls an organism's biological rhythm and regulates its physiological processes in response to external time cues. Most living organisms have their own time-keeping mechanism that is maintained by transcriptional-translational autoregulatory feedback loops involving several core clock genes, such as Period. Recent studies have found the relevance between the modulation of circadian oscillation and posttranscriptional modifications by microRNAs (miRNAs). However, there are limited studies on candidate miRNAs that regulate circadian oscillation. Here, we characterize the functions of novel miRNA-25 regulating circadian Period2 (Per2) expression. Using several in silico algorithms, we identified novel miR-25-3p that, together with miR-24-3p, targets the Per2 gene. Luciferase reporter assays validated that miR-25-3p and miR-24-3p repressed Per2 expression and confirmed their predicted binding sites in the $3^{\prime}$-untranslated region (UTR) of Per2 mRNA. Real-time bioluminescence analyses using Per2::Luc mouse embryonic fibroblasts confirmed that PER2 protein oscillation patterns were responsive to miR-25-3p and miR-24-3. The overexpression of miR-25-3p or miR-24-3p resulted in the dampening and period shortening of the PER2::LUC oscillation, while inhibition of either miRNA increased the relative amplitude of the PER2::LUC oscillation. Notably, endogenous miR-25-3p expression in the suprachiasmatic nucleus (SCN) showed no circadian rhythmicity, but the expression levels differed in various brain regions and peripheral tissues. These results suggest that the posttranscriptional regulation of miR-25-3p and miR-24-3p may differ according to Per2 gene expression in different tissue regions. In summary, we found that novel miR-25-3p was involved in fine-tuning circadian rhythmicity by regulating Per2 oscillation at the posttranscriptional level and that it functioned synergistically with miR-24-3p to affect Per2 oscillation.
\end{abstract}

\section{Introduction}

The circadian rhythm is one of the highly conserved features of the evolutionary process. Each species has its own biological rhythm governed by a master clock, the suprachiasmatic nucleus $(\mathrm{SCN})$ located in the hypothalamus in mammals or an equivalent structure that is reset in an approximate 24-h period according to the rotation of the Earth. This internal biological clock is essential for the

\footnotetext{
Correspondence: Kyungjin Kim (kyungjin@dgist.ac.kr)

'Department of Brain and Cognitive Sciences, Daegu Gyeongbuk Institute of Science and Technology (DGIST), Daegu 42988, Korea

${ }^{2}$ Department of Anatomy, Korea University College of Medicine, Seoul 02841, Korea
}

Full list of author information is available at the end of the article synchronization of bodily functions within an organism to maintain homeostasis, such as the sleep/wake cycle, metabolism, thermoregulation, and hormonal regulation ${ }^{1}$. Molecular clockwork involves genetically encoded autoregulatory feedback loops that maintain an approximately 24-h period of circadian oscillation. This self-sustainable biological clock machinery consists of positive and negative transcriptional-translational feedback loops ${ }^{2}$. The core loop of this molecular clock is driven by a heterodimeric transcriptional activator composed of two major clock genes: circadian locomotor output cycle kaput (CLOCK) and brain-muscle-arnt-like protein 1 (BMAL1) ${ }^{3}$. These heterodimers accelerate E-box-mediated transcription and increase the gene expression of negative regulators [such as 
Periods (PERs: PER1, PER2, and PER3) and Cryptochromes (CRYs: CRY1 and CRY2)] and circadian output genes. Expressed and dimerized PER:CRY represses the transcriptional activity of CLOCK:BMAL1, thereby downregulating their own gene transcription. As a result, these molecular feedback loops generate a 24-h circadian oscillation within an organism ${ }^{4,5}$.

In addition to the basic transcriptional-translational feedback loops, the oscillation of the molecular circadian clock is finely regulated by posttranscriptional and posttranslational modifications. In the present study, we investigated the posttranscriptional regulation of the Per2 gene, a negative regulator in the molecular clock machinery. Some small noncoding mature miRNAs of generally 21-25 nucleotides have been associated with the fine-tuning mechanisms of circadian oscillation ${ }^{6-8}$. The maturation of functional miRNAs is completed by endoribonuclease DICER in the cytoplasm, and each mature miRNA contains a seed sequence of 7 or 8 nucleotides that binds to its complementary region(s) on target mRNAs. A functional miRNA can interact with hundreds of target mRNAs to exert various levels of regulatory effect ${ }^{9}$, most by transcriptional repression in mammals ${ }^{10}$. A mature miRNAs bind to the $5^{\prime}$ - or $3^{\prime}$-untranslated region (UTR) of target mRNAs with the assistance of Argonaute (AGO) proteins and cause translational repression by preventing the initiation of translation ${ }^{11,12}$. Chen et al. $^{7}$ demonstrated the involvement of miRNAs in the expression of circadian molecular clock genes using DICER-deficient cells and conditional DICER-knockout mice. Their results revealed that the absence of miRNAs did not disrupt the generation of circadian rhythmicity; in contrast, it resulted in a significant shortening of the circadian period length ${ }^{13-15}$. Moreover, Chen et al. ${ }^{7}$ found that Per2 was the only affected core clock gene, with a slight elevation of its mRNA and a significant increase in PER2 protein levels. These findings support the previous understanding suggesting a role for the Per2 gene in circadian rhythm generation; i.e., PER2 is a negative regulator critical for modulating circadian rhythmicity in the approximate $24-\mathrm{h}$ period ${ }^{16}$.

However, the mechanism underlying the fine-tuning of circadian molecular oscillations via posttranscriptional modification by multiple miRNAs remains unknown. Here, we aimed to identify a miRNA that directly affects the expression of PER2 and determine the roles of multiple miRNAs in fine-tuning the molecular clockwork by comparing their impacts on temporal patterns of clock gene expression.

\section{Materials and methods Animals}

For circadian time point samples, 8-week-old C57BL/6J male wild-type (WT) mice were obtained, and Period2:: Luc knock-in (Per2::Luc KI) mice were sacrificed on postnatal days 7-9 to obtain samples for a brain slice culture. The mice were housed under a 12-h/12-h lightdark photoperiod at a constant temperature $\left(22-23^{\circ} \mathrm{C}\right)$. All procedures were conducted in compliance with the rules and regulations established by the Institutional Animal Care and Use Committee (IACUC) of Daegu Gyeongbuk Institute of Science and Technology (DGIST).

\section{Cell culture and transfection}

Per2::Luc knock in mouse embryonic fibroblasts (Per2:: Luc KI MEFs), wild-type MEFs, wild-type NIH3T3 and Per2-promoter-driven Luc mutant NIH3T3 cells were maintained in Dulbecco's modified Eagle's medium (DMEM, Gibco, USA) supplemented with $10 \%$ fetal bovine serum (Gibco) and 1\% antibiotic-antimycotic (AA, Gibco). The cells were cultured in a humidified incubator that was maintained at $37^{\circ} \mathrm{C}$ in a $5 \% \mathrm{CO}_{2}$ environment. The Per2::Luc KI MEFs were prepared from Per2::Luc KI transgenic mice carrying a luciferase reporter protein that was fused in-frame within an endogenous PER2 protein at the $\mathrm{C}$-terminus ${ }^{17}$. Reporter gene expression enabled realtime monitoring of PER2 protein dynamics with the intact 3'-UTR with Per2 miRNA-binding sites. Prior to transfection, cells were seeded in plates or dishes and allowed to grow for $24 \mathrm{~h}$. To overexpress or inhibit selected miRNAs, AccuTarget miRNA mimics and inhibitors (Bioneer, South Korea) were prepared and transfected using Lipofectamine 3000 transfection reagent (Invitrogen, USA) according to the manufacturer's instructions. Synthetic inhibitors for miR-24-3p and miR-25-3p were produced based on the miRbase sequence database (http://www.mirbase.org); the accession numbers were MIMAT0000219 and MIMAT0000652, respectively. For the control groups, a miR-control oligomer (Bioneer) was used. To examine the effects of these miRNAs on Per2 transcription, Per2::Luc KI MEFs were transfected with miR-control-, miR-24-3p-, or miR-25-3p-OE vectors (Applied Biological Materials, Canada) followed by dexamethasone (DEX) synchronization. Thirty hours after DEX synchronization, the cells were incubated with actinomycin D (at a final concentration of $1 \mu \mathrm{g} / \mathrm{ml}$ ) or DMSO and then harvested at 0 or $6 \mathrm{~h}$ after drug treatment for use in the downstream measurements.

\section{Organotypic slice culture}

An organotypic slice culture was prepared as described previously ${ }^{18}$, with some modifications. Briefly, the brains of neonatal mice (postnatal days 7-9) were obtained and rapidly placed in ice-cold Gey's balanced salt solution supplemented with $10 \mathrm{mM}$ HEPES and $30 \mathrm{mM}$ glucose, bubbled with $5 \%$ $\mathrm{CO}_{2}$ and $95 \% \mathrm{O}_{2}$. Coronal slices (400 $\mu \mathrm{m}$ thickness) were prepared using a vibratome (Leica, Nussloch, Germany). The slices were placed onto a culture membrane (Merck Millipore, USA) with $50 \%$ minimum essential medium, $25 \%$ Gey's 
balanced salt solution, $25 \%$ horse serum, $36 \mathrm{mM}$ glucose, and $100 \mathrm{U} / \mathrm{mL}$ antibiotic-antimycotic. The medium was replaced every 3 days before the experiments were performed. Then, the slice cultures were premeasured for PER2::LUC expression in recording medium supplemented with D-luciferin (Promega, USA) at a final concentration of $100 \mu \mathrm{M}$ using a real-time bioluminescence recording device (Kronos Dio, Japan). Each SCN slice culture was transduced with in-house generated lentiviral vector OE miR-control-GFP, miR-24-3pGFP, and/or miR-25-3p-GFP and incubated for 1 week. Then, the lentiviral transduction effect was recorded using the real-time bioluminescence device.

\section{DNA constructs and plasmids}

The full-length and truncated 3'-UTRs of Per2 were amplified from genomic DNA using the primers indicated. Each fragment was cloned into a plasmid with a pGL3 promoter (Promega). The following primers were used: Per2 3'-UTR forward for both fragments, 5'-AGA TCG CCG TGT AAT TCT AGA ATT AGA CGG TGC TCG GAA GA-3'; Per2 3'-UTR truncated reverse, 5'-TCT CAA GGG CAT CGG TCG ACT CCT CAC TGG TGA TGT CTC G-3'; and Per2 3'-UTR full-length reverse, 5'TCT CAA GGG CAT CGG TCG ACG ACA CAA GCA GTC ACA CAA-3'. The pGL3 promoter plasmid was $5 \mathrm{~kb}$, and the inserted $3^{\prime}$-UTRs were confirmed by gel electrophoresis based on their respective size of $6.8 \mathrm{~kb}$ (Per2 3'-UTR full-length) and $6 \mathrm{~kb}$ (Per2 3'-UTR truncated). The following mutations were introduced into the 3'-UTR of Per2 for the site-directed mutagenesis study: miR-24-3p, CUGAGCC $\rightarrow$ CCGCGCC ${ }^{7}$; miR-25-3p, GTGCAAU $\rightarrow$ GCGCGGU. The inserted fragments were sequenced (Cosmo Genetech, Korea) and validated using the NCBI BLASTN program (Fig. S3). miR-24-3p- and miR-25-3p-OE vectors and the control vector were purchased for the production of lentiviral vectors (Applied Biological Materials).

\section{Luciferase reporter assay}

Wild-type NIH3T3 fibroblasts were seeded in 96-well plates at $1 \times 10^{4}$ cell per well and then cotransfected with pRL-TK (50 ng per well), 3'-UTR of Per 2 inserted into a pGL3-promoter vector (150 ng per well) and miR-control oligomer or miR-24-3p and miR-25-3p mimics or inhibitors $(50 \mathrm{nM})$. After $48 \mathrm{~h}$ of transfection, luciferase activity levels were measured using a Dual-Glo luciferase assay system (Promega). Renilla luciferase activity was used to normalize the firefly luciferase activity.

\section{Real-time bioluminescence monitoring}

Per2::Luc KI MEFs and mutant NIH3T3 fibroblasts were plated in 35-mm dishes (Falcon, United States) at a $1.5 \times 10^{5}$ cell density. After $24 \mathrm{~h}$ of incubation, the cells were transfected with selected miRNAs $(50 \mathrm{nM})$ using Lipofectamine
3000 according to the manufacturer's protocol and incubated for $24 \mathrm{~h}$ (Invitrogen). The cells were synchronized by $200 \mathrm{nM}$ DEX treatment for $2 \mathrm{~h}$, and the bioluminescence was recorded for 5 days with a real-time bioluminescence device (Kronos Dio) in recording medium with $100 \mu \mathrm{M}$ D-luciferin (Promega). Light emissions were integrated for $1 \mathrm{~min}$ at intervals of 10 min using a dish-type wheeled luminometer. The raw data presented were normalized based on the first minimum bioluminescence point and detrended bioluminescence time series data were obtained by subtracting the 12-h moving average from the luminescence intensity with the background eliminated.

\section{RNA isolation and real-time quantitative PCR}

Total RNA, including small RNAs, was isolated using the Qiagen miRNeasy mini kit (Qiagen, Germany) according to the manufacturer's protocol. The isolated total RNA was reverse transcribed using an miScript II RT Kit (Qiagen) to quantify both the mRNAs and miRNAs in the total RNA sample. The miRNA expression profiles were analyzed by quantitative polymerase chain reaction (qPCR) using the miScript SYBR Green PCR kit (Qiagen) with designed primers (Qiagen), Mm_miR-24_1 miScript primer assay, Mm_miR-25_1 miScript primer assay and Hs_SNORD95_11 miScript primer assay for miR-24-3p, miR-25-3p and the internal control SnoRD95, respectively. The following primer sequences were used for mRNA quantification: Per2 up, 5'-ATCCCACGAACACCTCA TGA-3'; Per2 dn, 5'-CCCTGAGCTGTCCCTTTCTA-3'; Tbp up, 5'-GGG AGA ATC ATG GAC CAG AA-3'; Tbp dn, 5'-CCG TAA GGC ATC ATT GGA CT-3'.

\section{Western blotting}

Cells and tissue samples were washed with prechilled phosphate-buffered saline (PBS) and lysed in radioimmunoprecipitation assay buffer $[150 \mathrm{mM} \mathrm{NaCl}, 0.1 \%$ Triton X-100, $0.5 \%$ sodium deoxycholate, $0.1 \%$ sodium dodecyl sulfate, and $50 \mathrm{mM}$ Tris- $\mathrm{HCl}(\mathrm{pH} 8.0)]$ with Halt protease and phosphatase inhibitor cocktail (100x) (Thermo Scientific, USA) and sonicated in an ice bath. Supernatants were obtained, and total proteins were quantified using a Pierce BCA protein assay kit (Thermo Scientific). For each sample, $10 \mu \mathrm{g}$ of protein was loaded and separated by sodium dodecyl sulphate-polyacrylamide gel electrophoresis (7.5\% gel) and transferred to a polyvinylidene fluoride membrane (Merck Millipore). Immunoblot analyses were carried out with rabbit anti-PER2 polyclonal antibody (Santa Cruz Biotechnology, USA) and anti-beta-ACTIN-HRP (Santa Cruz Biotechnology).

\section{Immunohistochemistry and confocal imaging}

Transduced SCN slice cultures were fixated with a prechilled 4\% paraformaldehyde (PFA) solution and washed with PBS. For immunostaining, tissue samples were 
incubated with a DeepLabel antibody staining kit (Logos Biosystems, South Korea) permeabilization solution for $6 \mathrm{~h}$ and then labeled overnight with primary antibodies: rabbitanti-PER2 (1:500, Merck Millipore), mouse-anti-NeuN (1:500, Merck Millipore), and DAPI (1:10,000, Thermo Scientific) at $37^{\circ} \mathrm{C}$ with agitation. After washing with PBS three times, the appropriate secondary antibodies, donkey antirabbit-Alexa-594 and donkey anti-mouse-Alexa-647 (Invitrogen), were applied for $6 \mathrm{~h}$ at room temperature. The slice tissues were then imaged using a confocal LSM700 microscope (Carl Zeiss, Germany).

\section{Statistical analysis}

Statistical analyses were performed using Prism 8 (GraphPad Software, USA). For the luciferase reporter assay and real-time bioluminescence analyses, Cosinor software (https://www.circadian.org/softwar.html) was used for analyzing each period of detrended data format. To calculate the area under the curve, the data between the first and second nadirs from the raw data were analyzed using Prism 8 (Fig. S1). Student's $t$ test or one-way analysis of variance (ANOVA) was performed to assess the significance of intergroup differences. Significance was attributed for $p$ values $<0.05$. The results are represented as the means \pm standard error (SE). In general, the bioassays were run in duplicate and repeated (noted as " $n$ " in figure legends) for different occasions. The details of each statistical test used (statistics, significance levels, sample sizes and SEs) are described in each figure legend.

\section{Results}

Identification of miR-25-3p, a novel microRNA targeting the $3^{\prime}$-UTR of mouse Per2 mRNA

Three in silico databases, namely, TargetScan, miRDB, and DIANA microT-CDS, were screened for miRNAs that target the $3^{\prime}$-UTR of mouse circadian Per2 mRNA. miR-25-3p and miR-24-3p were selected as references based on their higher binding affinity for the $3^{\prime}$-UTR of Per 2 mRNA (their scores were approximately $90 \%$ or greater; data not shown). The complementary sequences of the miR-25-3p and miR-24-3p binding-sites on the $3^{\prime}-$ UTR of Per 2 mRNA were highly conserved among several vertebrates (Fig. 1a). After selecting miR-25-3p and miR24-3p, their predicted putative recognition sites on the $3^{\prime}$ UTR of Per 2 were identified using TargetScan. The complementary sequences of the seed miR-25-3p and miR-24-3p sequences were predicted to be on the $3^{\prime}$-UTR of Per2 mRNA at 1797-1804 and 1686-1692 nucleotides, respectively (Fig. 1b). Based on these predictions, two luciferase vectors were constructed: full-length $3^{\prime}$-UTR of Per2 (Per2 3'-UTR full-length) and 3'-UTR with truncated miR-25-3p and miR-24-3p binding sites (Per2 $3^{\prime}$-UTR truncated), as depicted in Fig. 1c. To determine whether miR-25-3p and miR-24-3p interact with their predicted complementary regions in the $3^{\prime}$-UTR of Per2, a dualluciferase assay was conducted with NIH3T3 fibroblasts, and pRL-TK was as a control for normalization. The relative luciferase activity levels of the Per2 3'-UTR full-length constructs were significantly attenuated by the miR-25-3p mimic or miR-24-3p mimic, compared to the effect of the miR-control oligomer treatment, while the expression of the truncated Per2 3 '-UTR construct was not affected (Fig. 1d). These results confirmed that miR-25-3p and miR-24-3p directly interact with the $3^{\prime}$ UTR of Per 2 and regulate gene expression.

miR-25-3p and miR-24-3p modulate Per2 gene expression at the posttranscriptional level

To measure the changes in miR-25-3p and miR-24-3p expression levels after transfecting each oligomer into WT MEFs at final concentrations of 25, 50, and $100 \mathrm{nM}$, real-time qPCR was performed (Fig. 2a). The dose-related fold increase over that of the control was exceptional. The large increase in miRNA levels after transfection was consistent with recent literature ${ }^{19,20}$. When transfecting the inhibitors, the target miRNAs were detected to be near zero, indicating the successful inhibition of the miRNAs of interest using their inhibitors. The expression levels of the target miRNAs compared to the that of the control group suggested that both primers, miRNA mimics, and inhibitors were specific to their targets. The optimal concentration of miRNA oligomers required to modulate the expression of PER2 protein was determined by Western blotting (Fig. 2b). Starting from the transfection dose at $50 \mathrm{nM}$, a decrease in PER2 protein levels was considered statistically significant.

To verify that miR-25-3p and miR-24-3p regulate Per2 after transcription, Per2::Luc KI MEFs were treated with actinomycin $\mathrm{D}$, a potent transcription inhibitor, after transfecting $0.5 \mu \mathrm{g}$ of miR-overexpressing $(\mathrm{OE})$ vectors (Fig. 2c). The MEFs were treated with actinomycin D for $30 \mathrm{~h}$ after dexamethasone synchronization and harvested at 0 and $6 \mathrm{~h}$ of actinomycin D treatment (Per2::Luc KI MEFs were harvested as indicated by the arrowheads in Fig. 2c) and assayed for Per2 mRNA levels. As expected, no differences in Per2 mRNA levels were found in the miR-25-3p- and miR-24-3p-OE MEFs before and after actinomycin D treatments compared to level in the control groups (Fig. 2d). Overall, miR-25-3p and miR-24-3p overexpression did not alter Per2 transcription but modulated the Per2 gene at the posttranscriptional level.

\section{miR-25-3p regulates circadian PER2 oscillation}

To determine the effect of miR-25-3p or miR-24-3p overexpression on rhythmic Per2 gene expression, Per2:: Luc KI MEFs were utilized for real-time bioluminescence analysis. Per2::Luc KI MEFs were constructed with a luciferase reporter inserted in frame at the end of exon 23 
a.

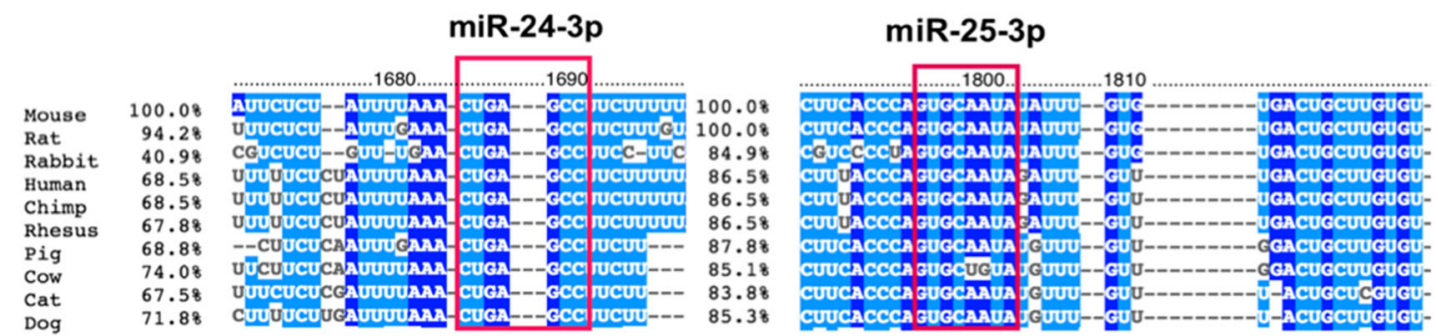

b.

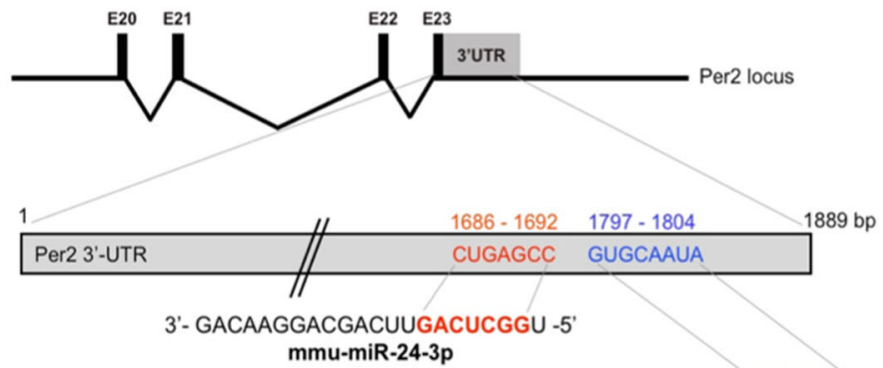

3'- AGUCUGGCUCUGUUCACGUUAC -5' mmu-miR-25-3p

c.

\section{pGL3-Per2-3'UTR full-length}

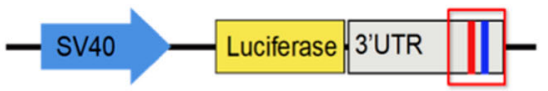

pGL3-Per2-3'UTR truncated

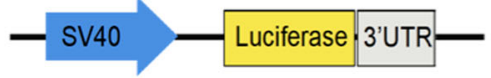

d.
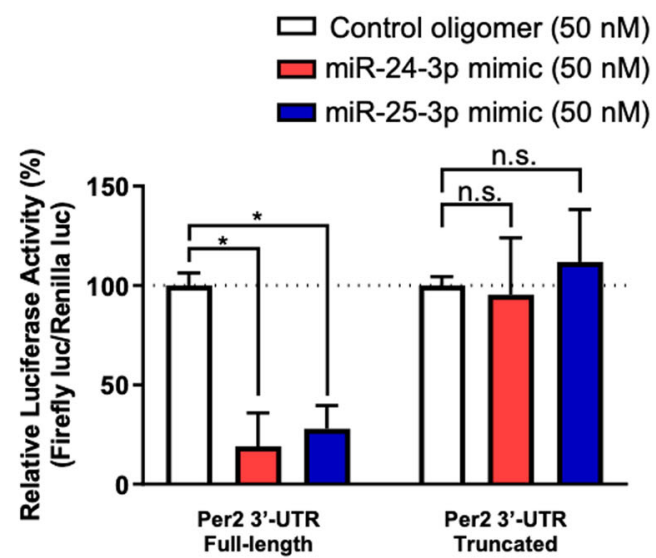

Fig. 1 Candidate microRNAs targeting the $\mathbf{3}^{\prime}$-untranslated (UTR) region of Period2 (Per2). a Conserved miR-24-3p and miR-25-3p binding sites on the $3^{\prime}$-UTR of Per2 in several vertebrates (indicated with red outlines). b Predicted binding sites of miR-24-3p (red) and miR-25-3p (blue) are illustrated on the $3^{\prime}-$ UTR of Per2, and the $3^{\prime}$-UTR targeting sequences of miR-24-3p and miR-25-3p are indicated. c Schematic of the constructed pGL3 vectors with binding sites on the $3^{\prime}$-UTR of Per2 for the full-length or truncated miR-24-3p and miR-25-3p. Predicted binding sites of miR-24-3p (red bar) and miR-25-3p (blue bar) are illustrated on the 3'-UTR of Per2. d NIH3T3 fibroblasts were cotransfected with pRL-TK and either a constructed pGL3 vector carrying a miR control oligomer $(50 \mathrm{nM})$, miR-24-3p mimic ( $50 \mathrm{nM}$, red), or miR-25-3p mimic ( $50 \mathrm{nM}$, blue). Data are presented as the means \pm SE $(n=4)$, and significance was assessed by Student's $t$ test $\left(^{*} p<0.01\right)$.

of the Per2 gene, followed by an endogenous $3^{\prime}$-UTR sequence $^{17}$. In summary, the Per2::Luc KI MEFs constituted an appropriate model system to study real-time Per2 expression as modulated by miR-25-3p or miR-24-3p in vitro. Interestingly, the Per2::Luc KI MEFs transfected with the miR-25-3p or miR-24-3p mimic showed not only shortened periods, by 71 and $13.5 \mathrm{~min}$, respectively, but also a significant dampening of their relative amplitudes, by $87.53 \%$ and $67.52 \%$, respectively (Fig. 3e). According to comparison studies of their nadirs and first peaks, the relative differences in phase advancement by both the miR-25-3p and miR-24-3p mimic treatments were increased (Fig. 3a, b). These results demonstrated that the miR-25-3p and miR-24-3p mimic treatments led to the dampening of PER2 expression and phase advancements at different levels. Synthetic miR inhibitors function as antagonists to their endogenous miRNAs by binding to the complementary sequences corresponding to the seed 
a.

miR-24-3p

miR-25-3p

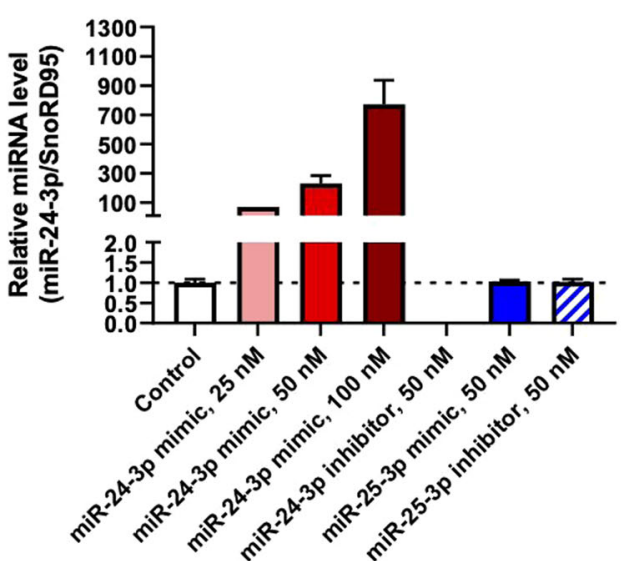

b.

miR-24-3p mimic
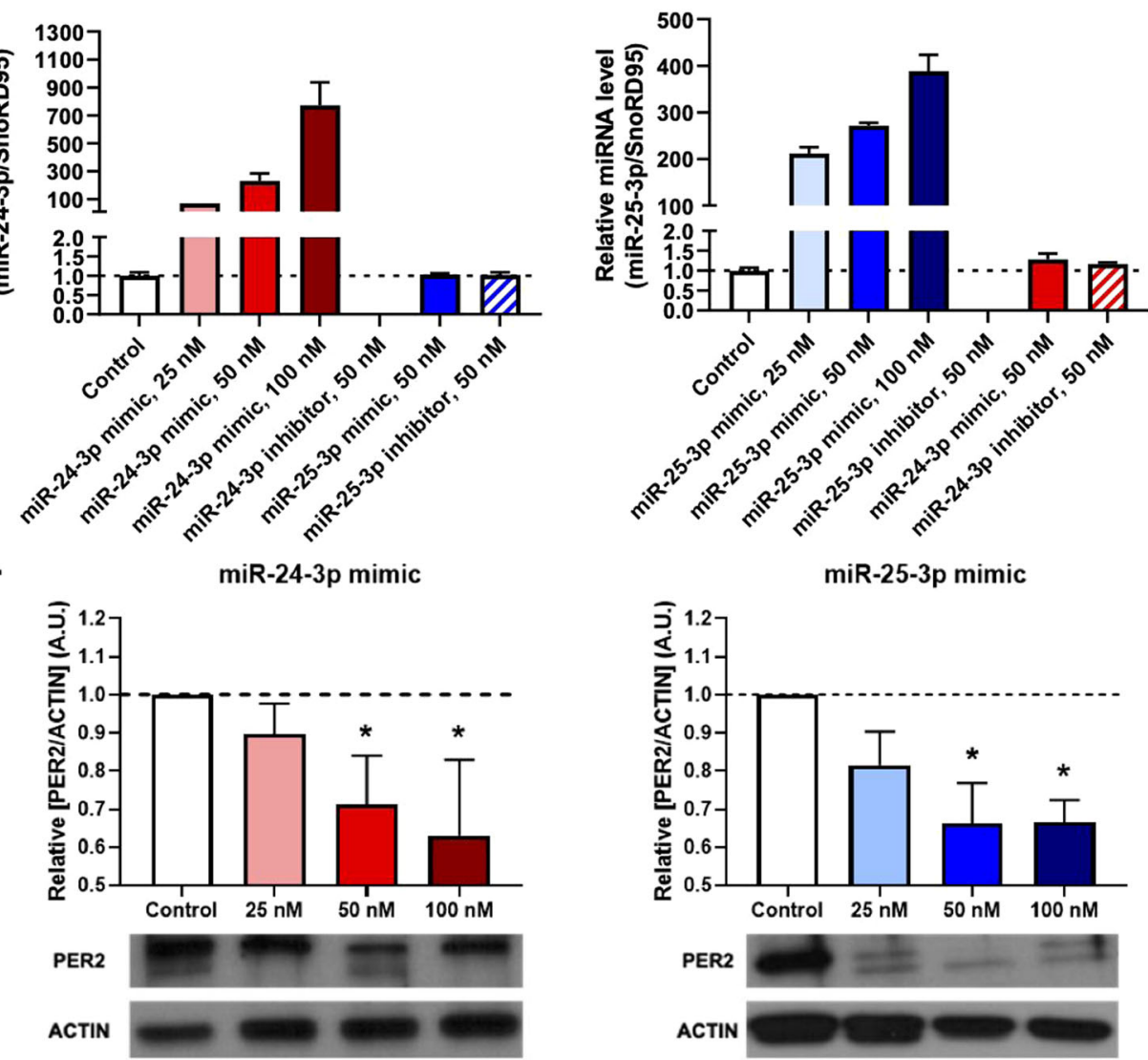

miR-25-3p mimic

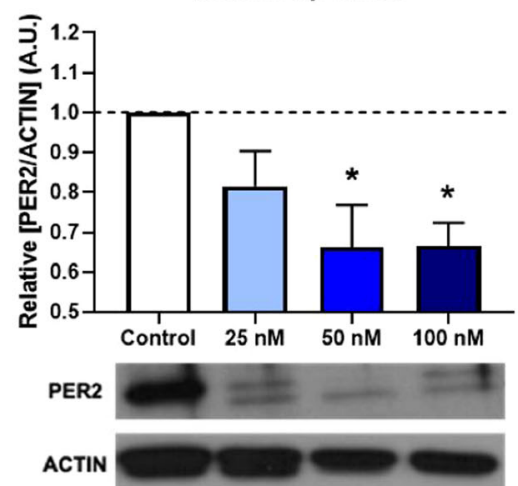

c.

miR-control

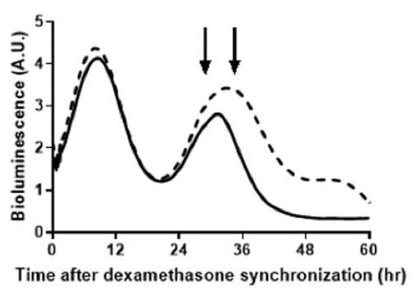

miR-24-3p OE

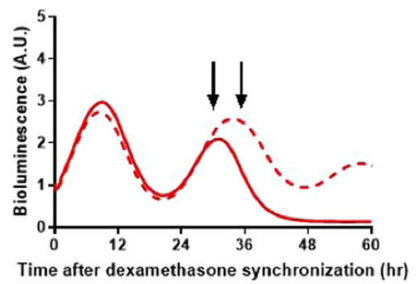

miR-25-3p OE

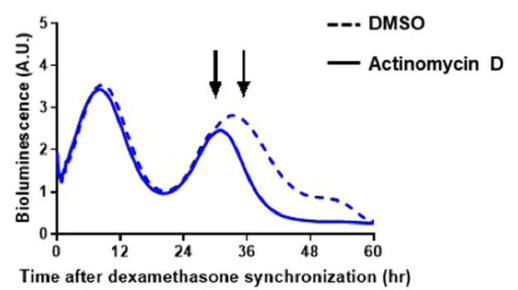

d.
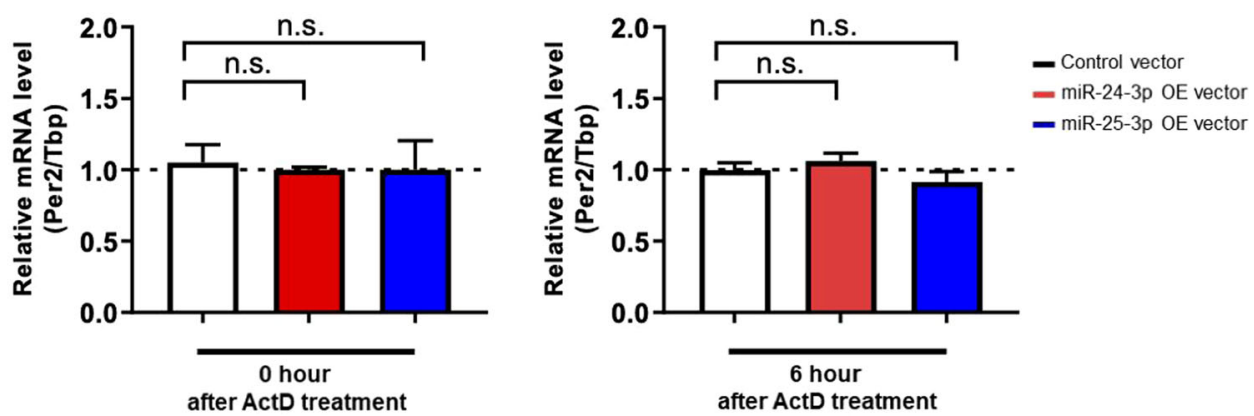

Fig. 2 (See legend on next page.) 
(see figure on previous page)

Fig. 2 miR-24-3p and miR-25-3p modulate Per2 gene expression at the posttranscriptional level. Wild-type mouse embryonic fibroblasts (WT MEFs) were transfected with miR-24-3p or miR-25-3p mimics in a dose-dependent manner (25,50, and $100 \mathrm{nM})$ and then assayed for the expression levels of $\mathbf{a}$ miR-24-3p and miR-25-3p using real-time qPCR $(n=4)$ and $\mathbf{b}$ quantified PER2 protein expression by Western blotting $(n=3)$. To determine the effects of miR-24-3p and miR-25-3p on Per2 transcription, Period2::Luc knock-in mouse embryonic fibroblasts (Per2::Luc KI MEFs) were transfected with $0.5 \mu \mathrm{g}$ of miR-24-3p- and miR-25-3p-overexpressing and control vectors, and then, actinomycin D (a potent transcription inhibitor) was added for $30 \mathrm{~h}$ after dexamethasone synchronization. c The results of real-time bioluminescence recordings are represented in raw data format after actinomycin D (solid line) and DMSO (dashed line) treatment. $\mathbf{d}$ Per2 mRNA was quantified after an additional 0 and $6 \mathrm{~h}$ of actinomycin D treatment (Per2::Luc KI MEFS were harvested as indicated by the arrowheads), and the data were normalized to that of the control vector $(n=4)$. Data are presented as the means $\pm \mathrm{SE}$, and the significance was assessed by one-way ANOVA, ${ }^{*} p<0.05$ compared to the control groups.

sequences in the target miRNAs ${ }^{21}$. To achieve loss of function, Per2::Luc KI MEFs were transfected with either a miR-25-3p or miR-24-3p inhibitor. As expected, the miR-25-3p and miR-24-3p inhibitors caused a significant delay in the circadian periods, by $30 \mathrm{~min}$ in both cases, and a significant increase in the relative amplitudes of PER2 expression, by $181.1 \%$ and $110.8 \%$, respectively (Fig. $3 \mathrm{c}-\mathrm{e})$. Similar to DICER-KO experiments ${ }^{7}$, the endogenous PER2 protein levels were significantly increased when the $3^{\prime}$-UTR of the Per2 mRNA-targeting miRNAs was blocked. Mutant Per2::Luc NIH3T3 fibroblasts transfected with miR-25-3p or miR-24-3p inhibitors were used for real-time bioluminescence analyses. In contrast to the Per2::Luc KI MEFs in the previous experiments, the mutant Per2::Luc fibroblast contained no Per2 3 '-UTR in the luciferase reporter construct, but the expression of the luciferase reporter was driven by the Per2 promoter. Despite transfecting the miR-25-3p and miR-24-3p inhibitors into mutant Per2::Luc NIH3T3 fibroblasts, no significant changes in the circadian periods or the amplitudes of PER2 expression were observed compared to the control group (Fig. 3f, g). Taken together, the data indicate that Per 2 expression can be altered through its $3^{\prime}$ UTR by either miR-25-3p or miR-24-3p.

miR-25-3p and miR-24-3p function in a synergistic manner by binding their seed sequences to the complementary target in $\mathbf{3}^{\prime}$-UTR of Per2

Herein, we evaluated the effect of the primary forms of miR-25-3p and miR-24-3p using miRNA-expressing vectors in a dose-dependent manner (Fig. 4a-f). Similar to the mature miRNA oligomer treatments, the CMV promoter-driven overexpression of miR-25-3p and miR24-3p resulted in a slight shortening of the PER2 period and dampening of the PER2 amplitude in a dosedependent manner. However, the different levels of PER2 oscillation altered by each OE miRNA suggest that there are specific optimal concentrations of miR-25-3p and miR-24-3p for modulating Per2 expression.

To validate the site-specific effects of miR-25-3p and miR-24-3p on Per 2 expression, we generated luciferase vectors driven by the Per 2 promoter with site-directed mutations in the $3^{\prime}$-UTR of Per2 corresponding to the seed sequences of miR-25-3p and/or miR-24-3p (Fig. 4g). Compared with that of the control vector and the wildtype $3^{\prime}$-UTR of Per2, the period of luciferase expression of the site-mutated $3^{\prime}$-UTR of Per2 was lengthened. The luciferase vector with double mutations in the miR-25-3pand miR-24-3p-binding sites showed a greater increase in luciferase amplitude compared to the $3^{\prime}$-UTR of Per2 vectors with single mutations of either the miR-25-3p- or miR-24-3p-binding site (Fig. 4h, i). This finding suggests that miR-25-3p and miR-24-3p have synergistic effects on Per2 expression and that each miRNA can function independently of each other.

\section{Endogenous miR-25-3p and miR-24-3p show no circadian oscillations in the slice cultures and brain tissues}

To explore the effects of miR-25-3p and miR-24-3p on the central master clock ex vivo, we cultured the SCN regions derived from Per2::Luc knock in mice at postnatal days 7-9, and the PER2::LUC oscillation was measured with real-time a bioluminescence recording. The SCN culture samples retained the endogenous neuronal networks within the SCN regions of the hypothalamus ${ }^{22}$. Plasmid vectors OE miR-25-3p or miR-24-3p (Fig. 4a-f) were used to generate the lentiviral vectors transduced into SCN slice cultures during a 1-week incubation period. To minimize experimental errors, the changes in PER2::LUC oscillations were compared for each SCN slice culture by comparing the measurement of the bioluminescence before lentiviral transduction and measurement of the recordings after the 1-week incubation with the miR-25-3p- and miR-24-3p-OE lentiviruses for transduction (Fig. 5a, left). Figure $5 \mathrm{~b}$ shows the results of the miR-25-3p-and/or miR-24-3p-OE lentiviral of vectors on PER2::LUC oscillations in the SCN slice cultures. Similar to the in vitro results, there were significant changes in the amplitudes of the PER2 levels when miR-25-3p and/or miR-24-3p were overexpressed (Fig. 5c). However, the differences in PER2 periods were not affected, as observed in the in vitro experiments (Fig. 5d).

To investigate the endogenous expression patterns of miR-25-3p and miR-24-3p, we quantified the mRNAs of Per2, Bmal1, miR-25-3p, and miR-24-3p in the SCN, a central clock region, and in the hippocampus, one of the 
a.

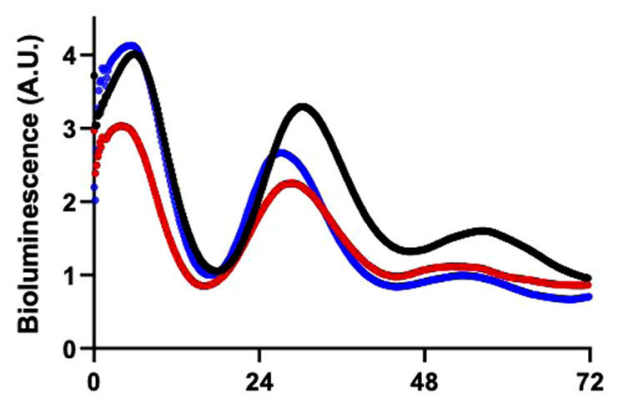

Time after dexamethasone synchronization (hr)

c.

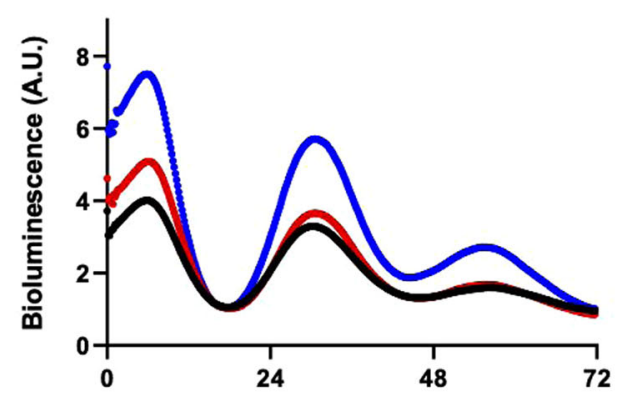

Time after dexamethasone synchronization (hr) b.

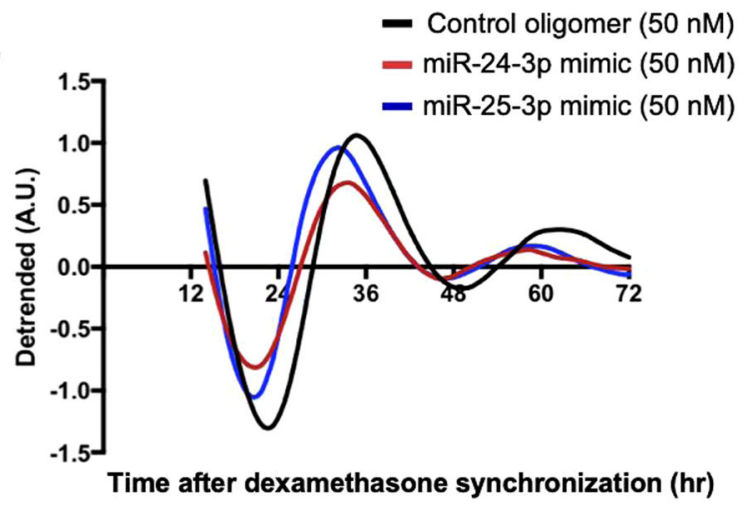

d.

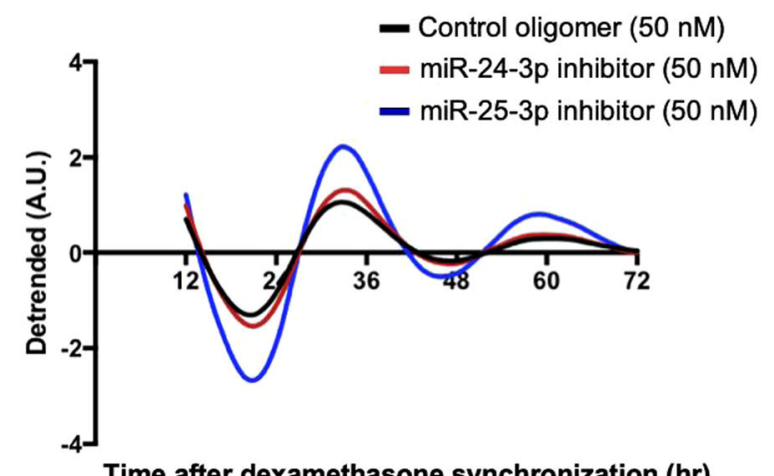

Time after dexamethasone synchronization (hr)

e.

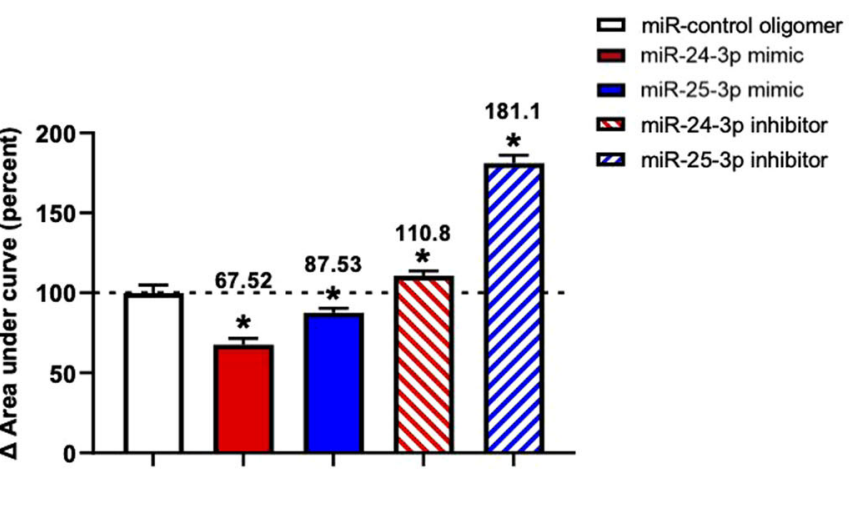

f.

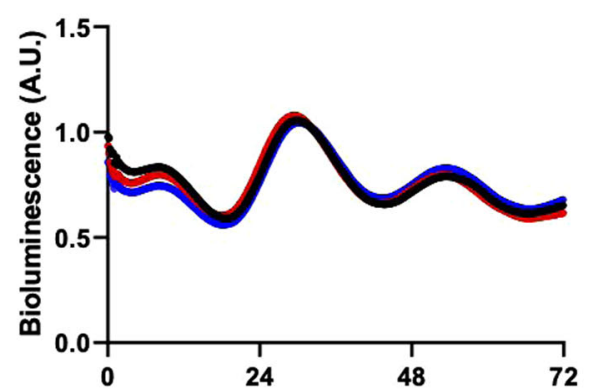

Time after dexamethasone synchronization (hr) g.

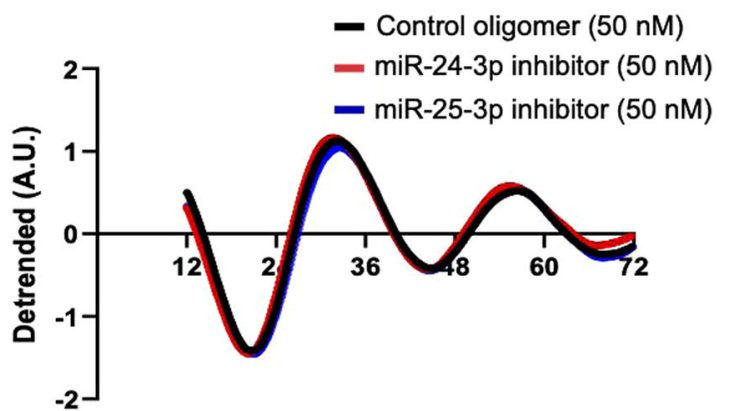

Time after dexamethasone synchronization (hr) 
(see figure on previous page)

Fig. 3 Effects of miR-24-3p or miR-25-3p on the oscillation of the PER2 protein. The results of real-time bioluminescence recordings are represented in raw and detrended data formats. a, b Per2::Luc KI MEFs were transfected with miR-24-3p or miR-25-3p mimics for gain-of-function studies. c, d For loss-of-function studies, miR-24-3p or miR-25-3p inhibitors were transfected into the Per2::Luc KI MEFs. Bioluminescence patterns were recorded for 3 days and normalized by the control group. e Relative periods and area under curve were calculated from the raw data, which is presented in bar graphs. $\mathbf{f}, \mathbf{g}$ Per2 promoter-driven luciferase (Per2pro-Luc) mutants were transfected into NIH3T3 fibroblasts with an miR-24-3p inhibitor, miR-25-3p inhibitor, or miR-control oligomer. Data are presented as the means $\pm \mathrm{SE}(n=4)$, and significance was assessed by one-way ANOVA, ${ }^{*} p<0.01$ compared to the control group.

local clock regions in the brain, at various circadian time points (Fig. 6a, b). Regardless of the clock gene mRNA oscillations in each brain region, neither miR-25-3p nor miR-24-3p showed rhythmic expression patterns, as they were expressed constitutively. Considering these nonrhythmic expression patterns of miR-25-3p and miR-24$3 p$, we further evaluated the expression levels of each miRNA in various brain regions and peripheral tissues at the fixed circadian time point, CT12. The expression levels of miR-24-3p were significantly higher in three regions, namely, the hippocampus, piriform cortex, and cerebellum (Fig. 6c), while miR-25-3p was more highly expressed in the cerebellum and kidney than in the other regions examined (Fig. 6d).

\section{Discussion}

Accumulation of the PER2 protein in the cytoplasm is necessary for its effective negative regulation of molecular clock genes. Previous studies have indicated the importance of miRNAs in regulating the expression of the circadian clock gene Per2 through posttranscriptional modification. Among the many candidate miRNAs targeting Per2 expression, miR-24-3p and miR-30a-5p were previously reported $^{7,13}$. In the present study, we demonstrated the modulatory function of a novel miRNA, miR-25-3p, in the regulation of Per2 at the posttranscriptional level. Overexpression of mature miR-25-3p led to a shortened period length and dampened amplitude of PER2 oscillation, while inhibition of miR-25-3p resulted in a lengthened period with a heightened amplitude of PER2 oscillation. Based on the predictions from the TargetScan 7.2 in silico databases and the functional validation made with mutant luciferase reporter assays, miR-25-3p was found to be involved in modulating the expression level of Per 2 by targeting its seedcomplimentary sequence on the $3^{\prime}$-UTR of Per 2 mRNA. The comparative study between miR-25-3p and miR-24-3p showed that miR-25-3p altered Per2 circadian rhythmicity, compared to that of miR-24-3p, to a significant degree.

miRNAs are known to interact with their seedcomplementary sequences on multiple target mRNAs and alter gene expression at the posttranscriptional level ${ }^{23,24}$. In addition, miRNAs also can induce differential effects on target mRNA expression via various binding affinities ${ }^{9,10}$. Our findings from the site-directed mutagenesis study showed that miR-25-3p and miR-24-3p can synergistically modulate the expression of PER2 oscillation, thereby explaining the multiplicity of miRNA function and their contributions in the fine-tuned regulation of circadian gene expression. Moreover, the results hint at the existence of various local clock oscillations in central and peripheral regions ${ }^{17,25,26}$, which may be generated by the posttranscriptional regulation by miRNAs. Our real-time qPCR results indicated different expression levels of miR-25-3p and miR-24-3p in various brain tissues and peripheral organs, which may have generated different modulatory effects on Per2 expression through various combinational effects of the miRNAs targeting Per2 expression. For instance, coherent circadian Per2 and Bmal1 anti-phasic gene expression was observed in both the SCN and hippocampus, showing different peak time points of Per2 in each region (Fig. 6), and PER2 protein expression was observed in the cerebellum and liver $^{13}$. These findings suggest plausible region-specific miR24-3p and miR-25-3p functions in modulating local Per2 oscillation, consequently producing individual local Per 2 oscillations in different tissue regions.

These modulatory functions of miRNAs have also been reported using DICER knockouts (KO) with loss of all the miRNAs in vitro and animal models ${ }^{7,27}$. Interestingly, these previous studies showed the persistent generation of Per2 gene oscillation at the molecular level and circadian locomotor activity in the DICER-KO animals even when the total miRNAs were genetically removed. Together, these findings support the suggestion that miRNAs are involved in the fine-tuning of circadian profiles rather than in generating circadian rhythmicity ${ }^{28}$. In addition, the results obtained by eliminating the specific modulatory functions of miRNAs on Per 2 expression by ablating endogenous Per $23^{\prime}$ UTR in an animal model (Per2::LucSV knock in transgenic animal) showed that there was a delayed phase shift of PER2 expression ${ }^{13}$. Notably, the phase delays of Per2::LucSV knock in ex vivo SCN cultures seemed to be governed by the heterozygosity of the Per $23^{\prime}$-UTR, because the homozygous mutants of the Per2 $3^{\prime}$-UTR showed a significant phase delay compared with the heterozygous mutants and wild-type. This result indicates that the posttranscriptional regulation of Per2 expression is also dominated by copy number variations. 
a.

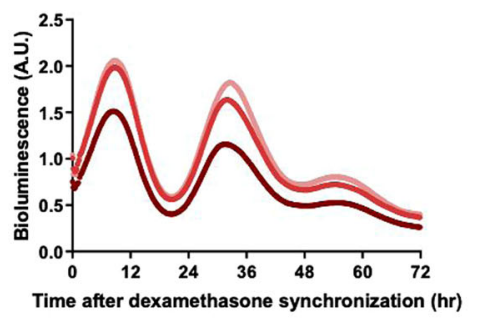

b.

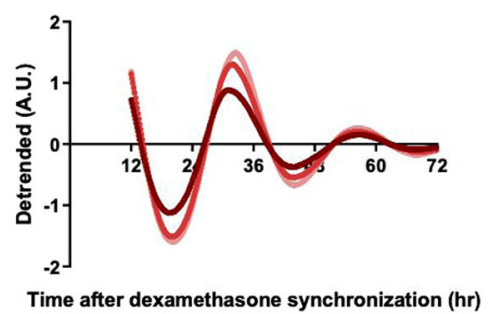

- miR-24-3p expressing vector $(0.10 \mu \mathrm{g})$

- miR-24-3p expressing vector $(0.25 \mu \mathrm{g})$
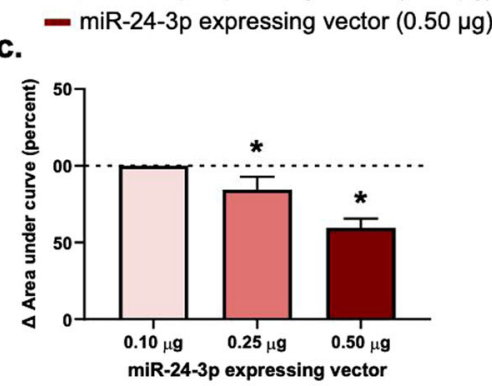

- miR-25-3p expressing vector $(0.10 \mu \mathrm{g})$

- miR-25-3p expressing vector $(0.25 \mu \mathrm{g})$

- miR-25-3p expressing vector $(0.50 \mu \mathrm{g})$

f.

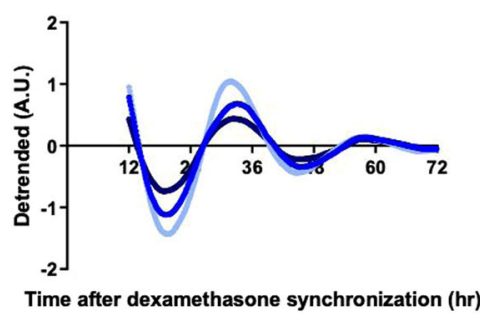

g.

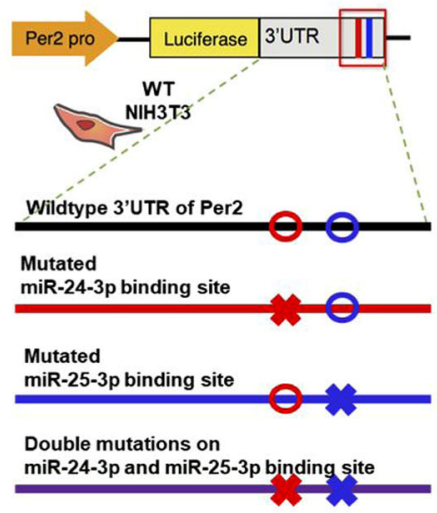

WT for miR-24: 5'-AAACTGAGCCTTCTT-3'

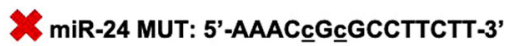

O WT for miR-25: 5'-CCCAGTGCAATATATT-3'

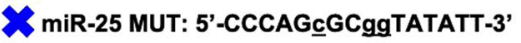

h.

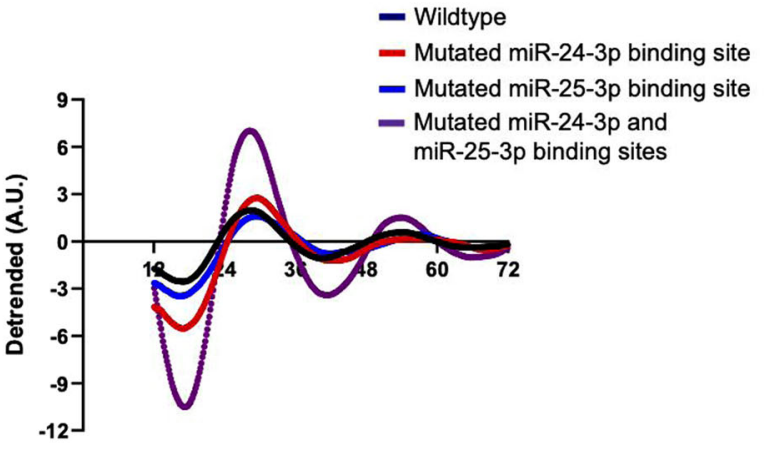

Time after dexamethasone synchronization (hr)

i.

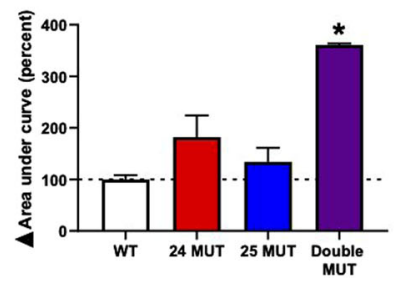

Fig. 4 miR-24-3p and miR-25-3p functioned in a dose-dependent manner and bind to their specific sites on the $3^{\prime}$-UTR of Per2. Real-time bioluminescence recordings of PER2::LUC oscillation in Per2::Luc KI MEFs that were transfected with $\mathbf{a}, \mathbf{b}$ miR-24-3p-overexpressing vectors or $\mathbf{d}$, e miR-25-3p-overexpressing vectors in a dose-dependent manner. $\mathbf{c}, \mathbf{f}$ The area under the curve was calculated from the raw data, which is presented in bar graphs. $\mathbf{g}$ Schematic for designed site-directed mutations of the miR-24-3p-and/or miR-25-3p binding sites on the 3'-UTR of Per2 in the pGL3LUC vector driven by the Per2 promoter. $\mathbf{h}$ Results of the real-time bioluminescence recordings of the transfected site-directed mutated luciferase vectors in the NIH3T3 wild-type fibroblasts and $\mathbf{i}$ the area under curve was calculated, which is presented in bar graphs. Data are presented as the means \pm SE $(n=3)$, and significance was assessed by one-way ANOVA, ${ }^{*} p<0.05$ compared to the control group. 

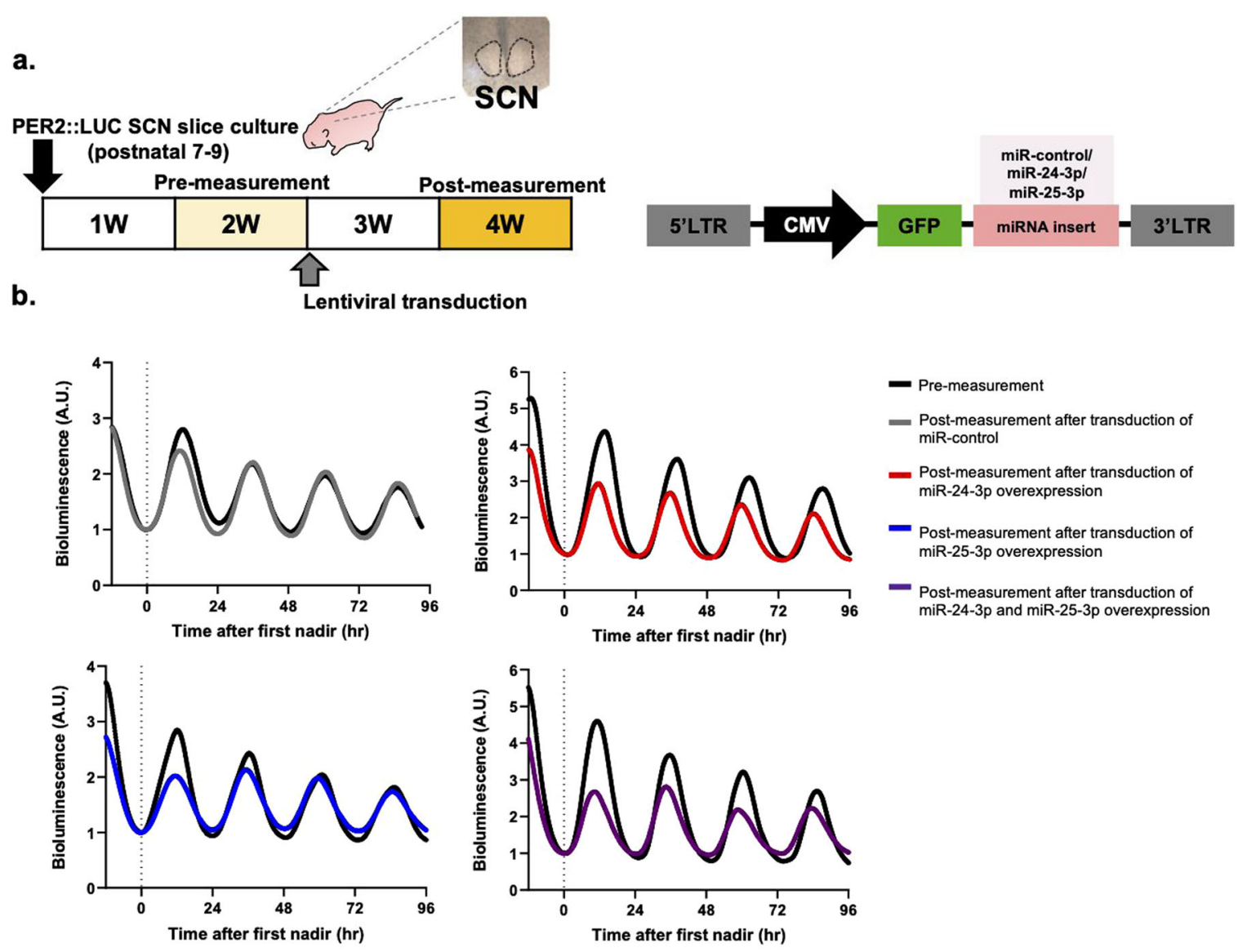

c.

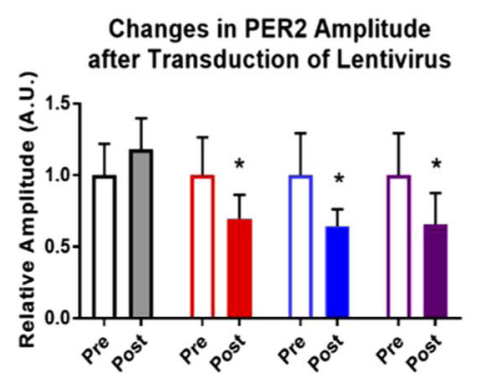

d.
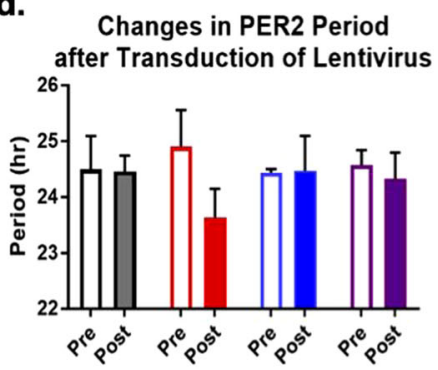

$\square$ LV-miR-Control

$\square$ LV-miR-24-3p

- LV-miR-25-3p

$\square$ LV-miR-24-3p + LV-miR-25-3p

Fig. 5 Transduction of lentivirus overexpressing miR-24-3p and/or miR-25-3p dampens the PER2::LUC rhythm in the neonatal SCN slice cultures obtained from the PER2::LUC knock in transgenic mice. a Experimental design for transducing CMV-promoter driven lentivirus-miRcontrol/miR-24-3p/miR-25-3p-GFP in neonatal suprachiasmatic nucleus (SCN) slice cultures. b Representative results of PER2::LUC oscillation measured by a real-time bioluminescence recording device. The bioluminescence patterns were aligned to the first nadir of data acquired to compare changes in the expression patterns of PER2::LUC in the pre- and postlentiviral transduced SCN slice cultures (experiments were performed three independent times, $n=3$ ). $\mathbf{c}$ Changes in PER2 amplitudes and periods are presented as the means \pm SE. Significance was assessed by Student's $t$ test, ${ }^{*} p<0.01$ compared to the prelentiviral transduction conditions.

This finding prompted us to speculate that the endogenous levels of miR-25-3p and miR-24-3p are crucial for their modulatory function and that this criterion is a possible explanation for the differential changes in the dose-dependent overexpression of miR-25-3p or miR24-3p (Fig. 3a-d) and the great dampening of the amplitude of PER2 oscillation achieved in ex vivo SCN
OE lentiviral miR-25-3p and/or miR-24-3p without altering the period lengths (Fig. 5). This outcome may be the result of a limited amount of effective miRNAs available for the posttranscriptional modification necessary to elicit changes in the Per2 period (Fig. S2). Moreover, these results indicate that there were potential compensatory factors within neuronal 

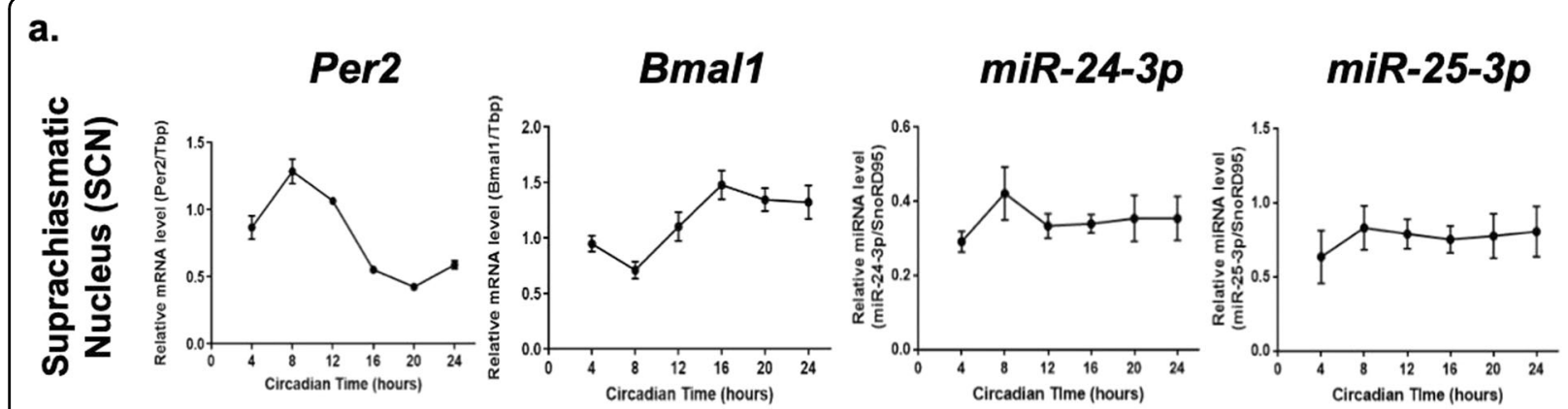

b.
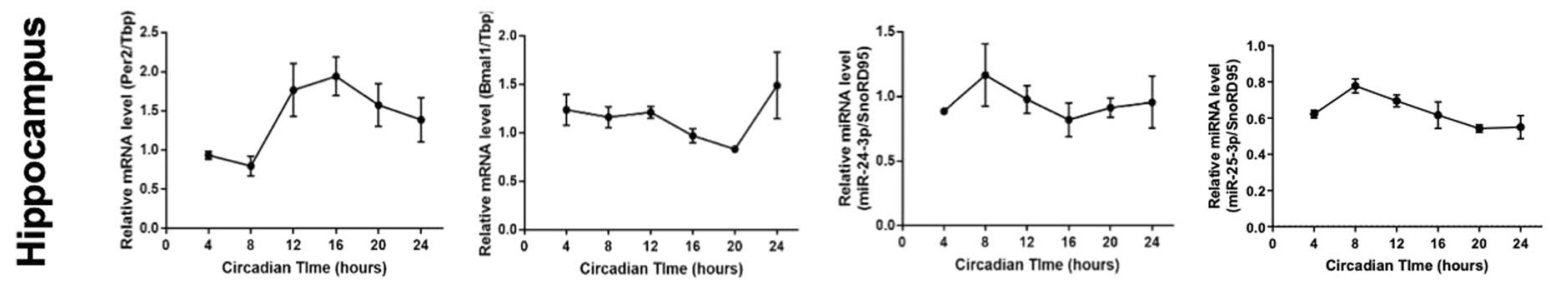

c.

d.
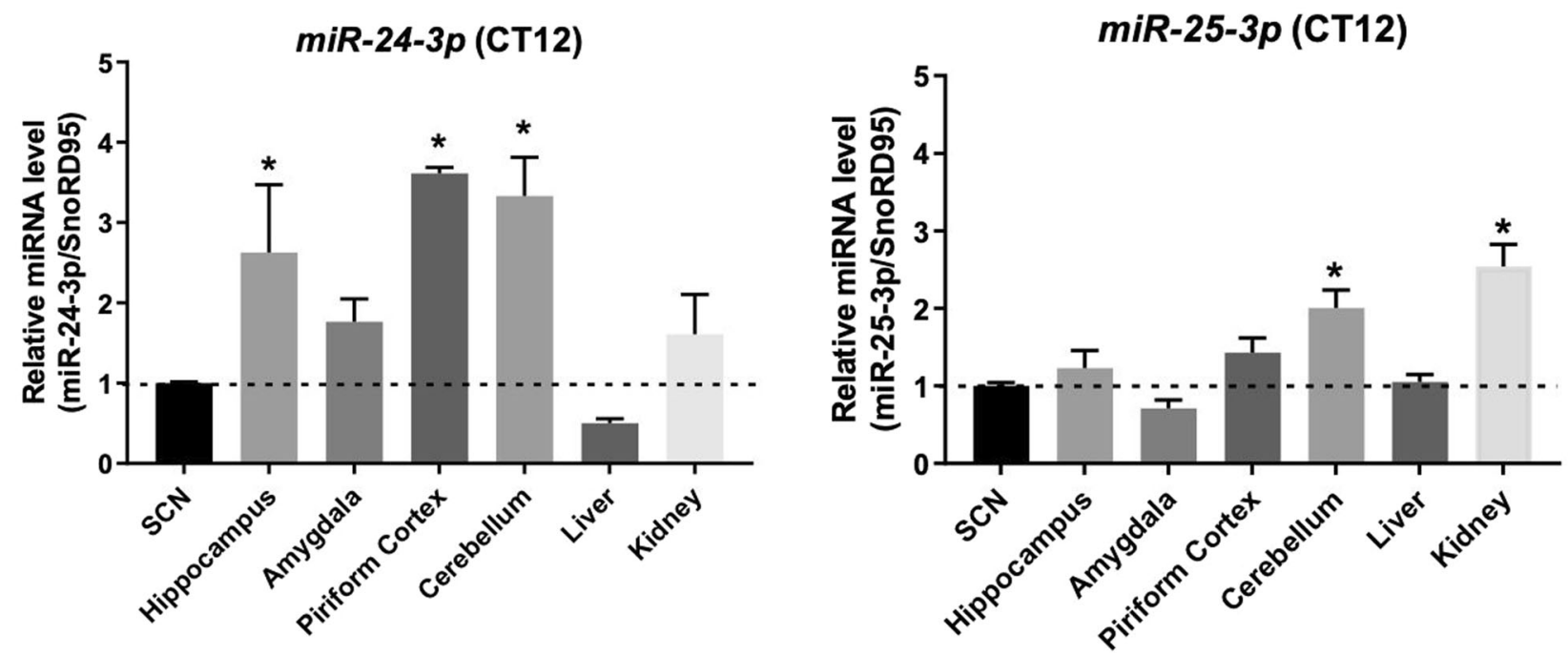

Fig. 6 Expression levels of miR-24-3p and miR-25-3p vary in brain regions and peripheral organs. WT mice housed under constant dark conditions for seven days were sacrificed at $\mathrm{CT} 04,08,12,16,20$, and $24 \mathrm{~h}$ for measurements of the indicated brain and peripheral tissues. Expression profiles of the circadian clock genes (Per2 and Bmal1 mRNAs), miR-24-3p, and miR-25-3p in the $\mathbf{a}$ SCN and $\mathbf{b}$ hippocampal brain tissues were obtained by real-time $q P C R$ with the relative quantification method. Expression levels of $\mathbf{c}$ miR-24-3p and $\mathbf{d}$ miR-25-3p in various brain and peripheral tissues were examined. CT12 tissue samples were used and normalized to the SCN miR-24-3p or miR-25-3p level. Data for the Per2 and Bmal1 mRNAs were normalized by the TATA-box binding protein (Tbp) housekeeping gene, while the miR-24-3p and miR-25-3p data were normalized by small nucleolar RNA, C/D Box 95 (SnoRD95). Error bars represent the means \pm SE of A.U. for each time point measured in three independent measurements. Significance was assessed by one-way ANOVA, ${ }^{*} p<0.05$ compared to the control group.

networks in the SCN culture that maintained the central circadian pace-making system. It is possible that an endogenous homeostatic system regulated the concentration of mature miR-25-3p and miR-24-3p in the SCN slice culture. To understand this, more extensive studies are required to comprehend the variations in miRNA effects on gene expression in vivo.

Recently, miRNAs have attracted considerable attention in the research field because of their functional connections to cancer. Interestingly, miR-25-3p and 
miR-24-3p are highly involved in tumorigenesis and have been extensively studied as biomarkers for cancer progression, as reported in recent literature ${ }^{29,30}$. Among genes with a high score predicting targeting by miR-25$3 \mathrm{p}$, B-cell translocation gene 2 (BTG2) is related to triple negative breast cancer, and miR-25-3p promotes the proliferation of breast cancer ${ }^{31}$. Moreover, miR-24$3 p$ targets the gene SOX7, which is involved in the progression of lung cancer ${ }^{32}$. These results may link the relationship between cancer progression and disrupted circadian rhythms as regulated by similar miRNAs, such as miR-25-3p and miR-24-3p.

The present study employed gain-of-function and lossof-function approach to understand the modulatory function of miR-25-3p on circadian Per2 gene expression. In addition to those of other previous studies, our results showed the synergistic effects of multiple miRNAs in the fine-tuning of Per 2 rhythmicity at approximately $24 \mathrm{~h}$, and this regulation might vary in different tissues. In conclusion, our study shows that miR-25-3p can modulate the expression of Per2 through posttranscriptional modification in addition to miR-24-3p and miR-30a-5 $p^{7,27}$. These miRNAs together function to fine-tune the circadian rhythm.

\section{Acknowledgements}

This work was supported by the Ministry of Science, ICT and Future Planning through the National Research Foundation of Korea (NRF-

2017R1A2A1A05001351 and NRF-2018M3C7A1022310), DGIST Start-up Fund Program (2020010055), KBRI Basic Research Program (20-BR-03-02), and an DGIST intramural grant (DGIST-20-RT-01).

\section{Author details}

'Department of Brain and Cognitive Sciences, Daegu Gyeongbuk Institute of Science and Technology (DGIST), Daegu 42988, Korea. ${ }^{2}$ Department of Anatomy, Korea University College of Medicine, Seoul 02841, Korea. ${ }^{3}$ Aging Neuroscience Research Group, Korea Brain Research Institute (KBRI), Daegu 41068, Korea

\section{Conflict of interest}

The authors declare that they have no conflict of interest.

\section{Publisher's note}

Springer Nature remains neutral with regard to jurisdictional claims in published maps and institutional affiliations.

Supplementary information accompanies this paper at https://doi.org/ 10.1038/s12276-020-00496-5.

Received: 17 December 2019 Revised: 22 June 2020 Accepted: 6 July 2020. Published online: 23 September 2020

\footnotetext{
References

1. Eckel-Mahan, K. \& Sassone-Corsi, P. Metabolism control by the circadian clock and vice versa. Nat. Struct. Mol. Biol. 16, 462-467 (2009).

2. Ko, C. H. \& Takahashi, J. S. Molecular components of the mammalian circadian clock. Hum. Mol. Genet. 15, R271-R277 (2006).

3. Hirayama, J. et al. CLOCK-mediated acetylation of BMAL1 controls circadian function. Nature 450, 1086-1091 (2007)

4. Balsalobre, A., Damiola, F. \& Schibler, U. A serum shock induces circadian gene expression in mammalian tissue culture cells. Cell 93, 929-937 (1998).
}

5. Gallego, M. \& Virshup, D. M. Post-translational modifications regulate the ticking of the circadian clock. Nat. Rev. Mol. Cell Biol. 8, 139-148 (2007).

6. Cheng, H. Y. et al. microRNA modulation of circadian-clock period and entrainment. Neuron 54, 813-829 (2007).

7. Chen, R., D'Alessandro, M. \& Lee, C. miRNAs are required for generating a time delay critical for the circadian oscillator. Curr. Biol. 23, 1959-1968 (2013).

8. Guo, H., Ingolia, N. T., Weissman, J. S. \& Bartel, D. P. Mammalian microRNAs predominantly act to decrease target mRNA levels. Nature $\mathbf{4 6 6}, 835-840$ (2010).

9. Filipowicz, W., Bhattacharyya, S. N. \& Sonenberg, N. Mechanisms of posttranscriptional regulation by microRNAs: are the answers in sight? Nat. Rev. Genet. 9, 102-114 (2008).

10. He, L. \& Hannon, G. J. microRNAs: small RNAs with a big role in gene regulation. Nat. Rev. Genet. 5, 522-531 (2004).

11. Kiriakidou, M. et al. An mRNA m7G cap binding-like motif within human Ago2 represses translation. Cell 129, 1141-1151 (2007).

12. Kwon, S. C. et al. Structure of human DROSHA. Cell 164, 81-90 (2016).

13. Yoo, S. H. et al. Period2 $3^{\prime}-U T R$ and microRNA-24 regulate circadian rhythms by repressing PERIOD2 protein accumulation. Proc. Natl Acad. Sci. USA 114, E8855-E8864 (2017).

14. Crosby, P. et al. Insulin/GF-1 drives PERIOD synthesis to entrain circadian rhythms with feeding time. Cell 177, 1-14 (2019).

15. Luo, W. \& Sehgal, A. Regulation of circadian behavioral output via a microRNAJAK/STAT circuit. Cell 148, 765-779 (2012).

16. Meng, Q. J. et al. Setting clock speed in mammals: the CK1 epsilon tau mutation in mice accelerates circadian pacemakers by selectively destabilizing PERIOD proteins. Neuron 58, 78-88 (2008).

17. Yoo, S. H. et al. PERIOD2::LUCIFERASE real-time reporting of circadian dynamics reveals persistent circadian oscillations in mouse peripheral tissues. Proc. Natl Acad. Sci. USA 101, 5339-5346 (2004).

18. Choe, H. K. et al. Synchronous activation of gonadotropin-releasing hormone gene transcription and secretion by pulsatile kisspeptin stimulation. Proc. Nat Acad. Sci. USA 110, 5677-5682 (2013).

19. Takeshita, H. et al. XB130, a new adaptor protein, regulates expression of tumor suppressive microRNAs in cancer cells. PLoS ONE 8, e59057 (2013).

20. Genz, B. et al. Overexpression of miRNA-25-3p inhibits Notch1 signaling and TGF-beta-induced collagen expression in hepatic stellate cells. Sci. Rep. 9, 8541 (2019).

21. Stenvang, J., Petri, A., Lindow, M., Obad, S. \& Kauppinen, S. Inhibition of microRNA function by antimiR oligonucleotides. Silence 3, 1-17 (2012).

22. Thompson, A. A., Walmsley, S. R. \& Whyte, M. K. A local circadian clock calls time on lung inflammation. Nat. Med. 20, 809-811 (2014).

23. Kojima, S., Shingle, D. L. \& Green, C. B. Post-transcriptional control of circadian rhythms. J. Cell Sci. 124, 311-320 (2011).

24. Lim, C. \& Allada, R. Emerging roles for post-transcriptional regulation in circadian clocks. Nat. Neurosci. 16, 1544-1550 (2013).

25. Kornmann, B., Schaad, O., Bujard, H., Takahashi, J. S. \& Schibler, U. System-driven and oscillator-dependent circadian transcription in mice with a conditionally active liver clock. PLoS Biol. 5, 179-189 (2007).

26. Hastings, M., O'Neill, J. S. \& Maywood, E. S. Circadian clocks: regulators of endocrine and metabolic rhythms. J. Endocrinol. 195, 187-198 (2007).

27. Du, N. H., Arpat, A. B., De Matos, M. \& Gatfield, D. MicroRNAs shape circadian hepatic gene expression on a transcriptome-wide scale. elife $\mathbf{3}$, e02510 (2014).

28. Yates, L. A., Norbury, C. J. \& Gilbert, R. J. The long and short of microRNA. Cell 153, 516-519 (2013)

29. Ogata-Kawata, H. et al. Circulating exosomal microRNAs as biomarkers of colon cancer. PLoS ONE 9, e92921 (2014).

30. Khodadadi-Jamayran, A. et al. Prognostic role of elevated mir-24-3p in breast cancer and its association with the metastatic process. Oncotarget $\mathbf{9}$ 12868-12878 (2018).

31. Chen, H., Pan, H., Qian, Y., Zhou, W. \& Liu, X. miR-25-3p promotes the proliferation of triple negative breast cancer by targeting BTG2. Mol. Cancer 17, 4-4 (2018).

32. Yan, $\mathrm{L}$. et al. miR-24-3p promotes cell migration and proliferation in lung cancer by targeting SOX7. J. Cell. Biochem. 119, 3989-3998 (2018). 